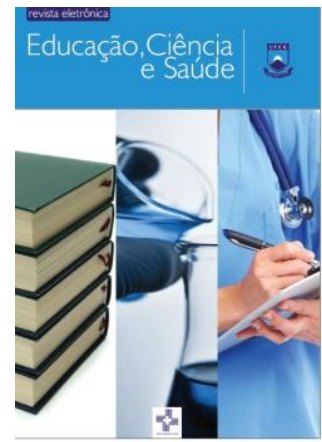

EDUCAÇÃO CIÊNCIA E SAÚDE

http://dx.doi.org/10.20438/ecs.v5i1.143

\title{
MICRORGANISMOS ISOLADOS DE UROCULTURAS EM UM HOSPITAL UNIVERSITÁRIO DO ESTADO DA PARAÍBA, BRASIL
}

\author{
Alex Carneiro da Cunha Nóbrega Junior ${ }^{1}$, Maria Franncielly Simões de Morais ${ }^{2}$, \\ Ana Carolina Pessoa Moreira ${ }^{3}$, ${ }^{\star}$ Egberto Santos Carmo ${ }^{4}$
}

\footnotetext{
${ }^{1}$ Especialização em farmácia clínica e prescrição farmacêutica, ICTQ, João Pessoa-PB.

${ }^{2}$ Curso de Bacharelado em Farmácia, Unidade Acadêmica de Saúde, Universidade Federal de Campina Grande, Cuité-PB, Brasil.

${ }^{3}$ Faculdade Maurício de Nassau, Vitória da Conquista-BA.

${ }^{4}$ Profo Unidade Acadêmica de Saúde, Universidade Federal de Campina Grande, Cuité, PB, Brasil.

`Email para correspondência: egberto santos@yahoo.com.br
}

\begin{abstract}
Resumo
A infecção do trato urinário (ITU) é a segunda mais comum na população, especialmente entre as adquiridas em ambiente hospitalar. O objetivo foi avaliar a prevalência microbiana em uroculturas, descrevendo características dos pacientes e o perfil de sensibilidade a antimicrobianos das espécies mais comuns. Pesquisa realizada nas fichas de avaliação de pacientes com suspeita de ITU de um Hospital Universitário em Campina Grande-PB, entre julho e dezembro de 2012, com aprovação do Comitê de ética, CAAE Nr. 17908413.6.0000.5182 e Parecer Nr. 520.951. Das 554 uroculturas realizadas, $124(22,4 \%)$ mostraram-se positivas para crescimento microbiano. No ambulatório, a Escherichia coli apresentou maior prevalência $(61,8 \%)$, seguida por Klebsiella pneumoniae $(8,8 \%)$. Com relação às uroculturas dos pacientes internos, houve uma inversão com $K$. pneumoniae $(27,8 \%)$ seguida da $E$. coli $(26,7 \%)$. A maior resistência de $E$. coli foi a ampicilina (50\%) no ambulatório. O sexo feminino foi o mais acometido por ITU, elevando-se os casos com o avançar da idade, principalmente após 50 anos. O conhecimento da epidemiologia das infecções do trato urinário e a implantação de protocolos terapêuticos para utilização de antimicrobianos, juntamente com os resultados do antibiograma, pode permitir uma redução da resistência aos agentes antimicrobianos.
\end{abstract}

Palavras-chave: infecção, Escherichia coli e Klebsiella pneumoniae.

\section{Abstract / resumen / résumé}

Urinary tract infection (UTI) is the second most common in the population, especially among those acquired in a hospital environment. The objective was to evaluate the microbial prevalence in urocultures, describing the characteristics of the patients and the antimicrobial susceptibility profile of the most common species detected at the University Hospital Alcides Carneiro (HUAC), in Campina Grande- 
PB from July to December 2012. Ethics Committee, case No. 17908413.6.0000.5182 and Technical opinion No. 520.951. Of the 554 urocultures performed, 124 (22.4\%) were positive for microbial growth. In the outpatient clinic, Escherichia coli presented a higher prevalence $(61.8 \%)$, followed by Klebsiella pneumoniae (8.8\%). Regarding the urocultures of the internal patients, there was an inversion with $K$. pneumoniae $(27.8 \%)$ followed by $E$. coli $(26.7 \%)$. The highest resistance of $E$. coli was ampicillin $(50 \%)$ in the outpatient clinic. The female sex was the most affected by UTI, with cases increasing with age, especially after 50 years. Knowledge of the epidemiology of urinary tract infections and the implementation of therapeutic protocols for antimicrobial use, along with antibiogram results, may allow a reduction in resistance to antimicrobial agents.

Keywords: infection, Escherichia coli and Klebsiella pneumoniae.

\section{Introdução}

O trato urinário, geralmente, se mantem estéril, em condições fisiológicas, especialmente pela osmolalidade, $\mathrm{pH}$ baixo e a alta concentração de ureia que inibem o crescimento de muitas espécies de bactérias. Porém quando algum microrganismo entra nessas vias urinárias, depende de fatores como tamanho do inóculo, imunidade do hospedeiro, além do uso de sondas vesicais, pode determinar o surgimento de uma infecção do trato urinário (KONEMAN et al., 2008; CYRINO; STUCHI, 2016).

Tais infecções são classificadas quanto à presença de fatores predisponentes ou agravantes, de origem hospitalar ou comunitária. A ITU não complicada ocorre em paciente com estrutura e função do trato urinário normal, preferencialmente em mulheres jovens, sexualmente ativas e adquirida fora de ambiente hospitalar. As consideradas complicadas estão associadas a alguma anormalidade estrutural ou funcional do processo de diurese, presença de cálculos renais ou prostáticos, incluindo as de causa obstrutiva, metabólicas e uso de cateter (NEGROMONTE et al., 2015; HACHUL et al., 2015).

A etiologia das ITUs varia com o gênero, idade, estado geral do paciente, uso de antimicrobianos e a obtenção dentro ou fora do hospital. Quando a infecção é adquirida no ambiente hospitalar, os agentes etiológicos são bastante diversificados, predominando as Enterobactérias, com ligeira redução na frequência de E. coli e um crescimento de Proteus sp, Pseudomonas aeruginosa, Klebsiella sp., Enterobacter sp., Enterococcus faecalis e de fungos, com destaque para Candida sp (KSYCKI; NAMIAS, 2009; PELEG; HOOPER, 2010; DAS et al., 2017).

Laboratorialmente a cultura representa um importante aliado no diagnóstico de uma ITU. Havendo crescimento microbiano nos meios de 
cultura usuais, este deve ser avaliado quanto à possibilidade de constituir microbiota normal ou, se há suspeita de microrganismo potencialmente patogênico, proceder ao processo de identificação através das provas bioquímicas de identificação. Deve-se, ao menos, constar o gênero de patógenos potenciais, embora com o advento dos equipamentos de automação, a identificação em gênero e espécie tenha se transformado em prática diária (KONEMAN et al., 2008; ALLGAYER; SCHIRMER; CASTELAN, 2015; MIRANDA et al., 2016).

A realização do antibiograma permite a detecção do antimicrobiano mais eficaz in vitro. A escolha dos antimicrobianos a serem utilizados no teste é baseada em espectro de ação, propriedades farmacocinéticas, toxicidade, eficácia e disponibilidade (COSTA, 2009).

A resistência aos antimicrobianos tem aumentado no Brasil e no mundo, gerando uma necessidade crescente de definição do perfil de suscetibilidade dos agentes microbianos patogênicos mais prevalentes e do mecanismo de transmissão/aquisição de resistência (ROSA et al., 2017).

As infecções do trato urinário representam grande preocupação no ambiente hospitalar, pois estão inseridas entre as infecções nosocomiais mais frequentes. A ausência de dados para realização de um tratamento específico, tanto relacionados ao agente etiológico, como aos resultados do antibiograma, podem acarretar em uma terapia empírica inadequada, e, consequentemente, em falha terapêutica, permitindo assim o surgimento de infecções recorrentes. Isto pode ser evitado pela avaliação criteriosa do quadro clínico do paciente associada ao resultado da urocultura e do antibiograma.

Diante do exposto, este estudo objetivou verificar, através de resultados de uroculturas de origem comunitária e hospitalar, de registros realizados no Hospital Universitário Alcides Carneiro, em Campina Grande - PB, a prevalência microbiana em uroculturas, descrevendo características dos pacientes e o perfil de sensibilidade a antimicrobianos das espécies mais comuns. 


\section{Metodologia}

Pesquisa exploratória, descritiva e analítica, com abordagem quantitativa, no qual os dados foram descritos e sintetizados, fazendo o uso de valores em porcentagem (POLIT; BECK; HUNGLER, 2004).

O local da pesquisa foi o setor de microbiologia do Laboratório de Análises Clínicas do Hospital Universitário Alcides Carneiro (HUAC) da Universidade Federal de Campina Grande (UFCG).

A pesquisa teve por base informações registradas em fichas de avaliação de pacientes com suspeita clínica de infecção do trato urinário, em que as amostras foram submetidas à urocultura no Setor de Microbiologia do Laboratório de Análises Clínicas do Hospital Universitário Alcides Carneiro, em Campina Grande-PB, no período de julho a dezembro de 2012.

A população foi composta por todos os resultados de exames microbiológicos registrados no laboratório, sendo a amostra composta por todos os resultados de exames de urocultura no período referido, resultantes da demanda de pacientes internos e ambulatoriais atendidos no HUAC.

Foi utilizada uma planilha de Excel para notação de dados como sexo, idade, origem do paciente, entre outros.

Foram incluídas no estudo todas as fichas de notificação de infecção urinária, as quais continham todos os dados necessários para o estudo preenchidos completamente, como idade, sexo e resultado de antibiograma. Foram excluídas da pesquisa todas as fichas que não fossem referentes à infecção do trato urinário.

O estudo foi desenvolvido após aprovação do Comitê de Ética em Pesquisa com seres humanos, sob CAEE: 17908413.6.0000.5182 e Parecer Nr. 520.951, conforme Diretrizes e Normas regulamentares de pesquisa envolvendo seres humanos, da Resolução 466/12 do Conselho Nacional de Saúde.

\section{Resultados e discussão}

No período de julho a dezembro de 2012 foram realizadas 554 uroculturas no Laboratório de Microbiologia do referido Hospital. Destas, 124 (22,4\%) mostraram-se positivas para crescimento microbiano. 
Foram observadas muitas culturas com resultados negativos e para isso, existem algumas justificativas, as quais podem explicar à Síndrome Uretral ou Síndrome Piúria-Disúria ou "Abacteriúria sintomática". Dentre elas, infecções por germes fastidiosos ou não habituais como Chlamydia trachomatis, Neisseria gonorrhoeae, Mycoplasma e Trichomonas vaginalis. Amostras urinárias obtidas durante tratamento ou em uso de agentes antissépticos também podem justificar elevado número de culturas de urina negativas (HEILBERG; SCHOR, 2003).

Com a relação à prevalência de microrganismos identificados no laboratório de microbiologia do referido hospital, tanto pra infecções urinárias ambulatoriais quanto nosocomiais, duas bactérias destacaram-se: Escherichia coli e Klebsiella pneumoniae. Contudo, E. coli foi responsável pela grande maioria dos casos $(61,8 \%)$ de origem ambulatorial (figura 1$)$, seguida por $K$. pneumoniae $(8,8 \%)$, enquanto que esta última bactéria aparece percentualmente um pouco maior $(27,8 \%)$, seguida de E. coli $(26,7 \%)$ nos casos de ITU nosocomiais (figura 2).

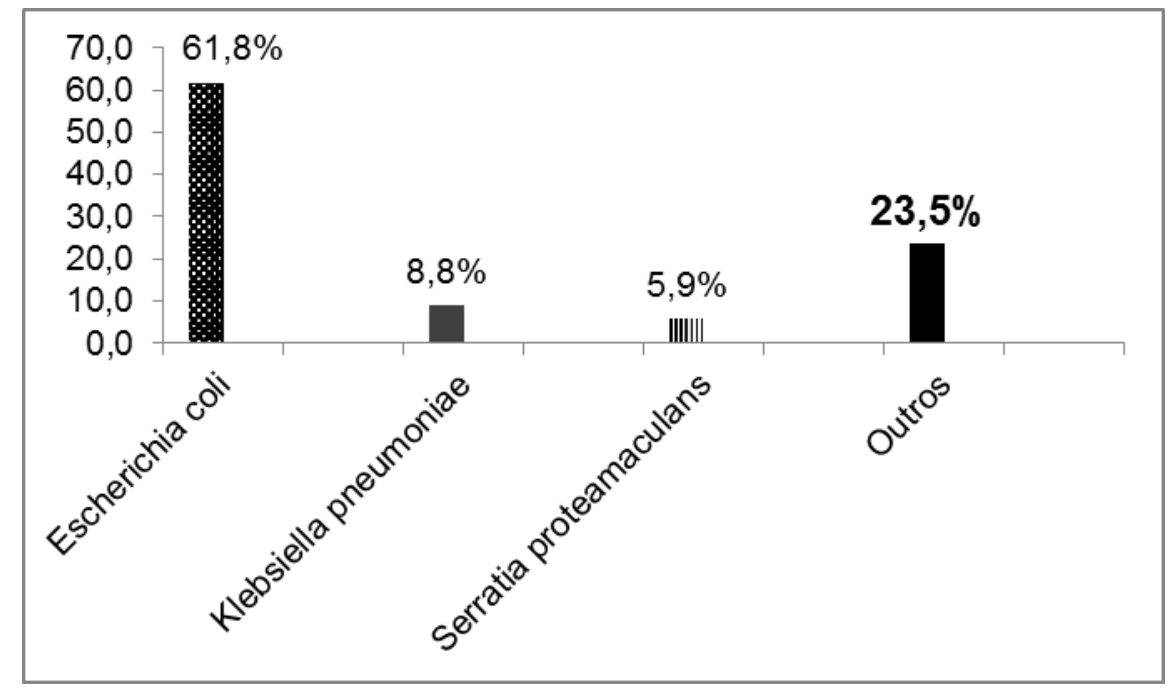

Figura 1 - Prevalência de microrganismos isolados em exames de urocultura ambulatorial no HUAC.

Fonte: Pesquisadores 


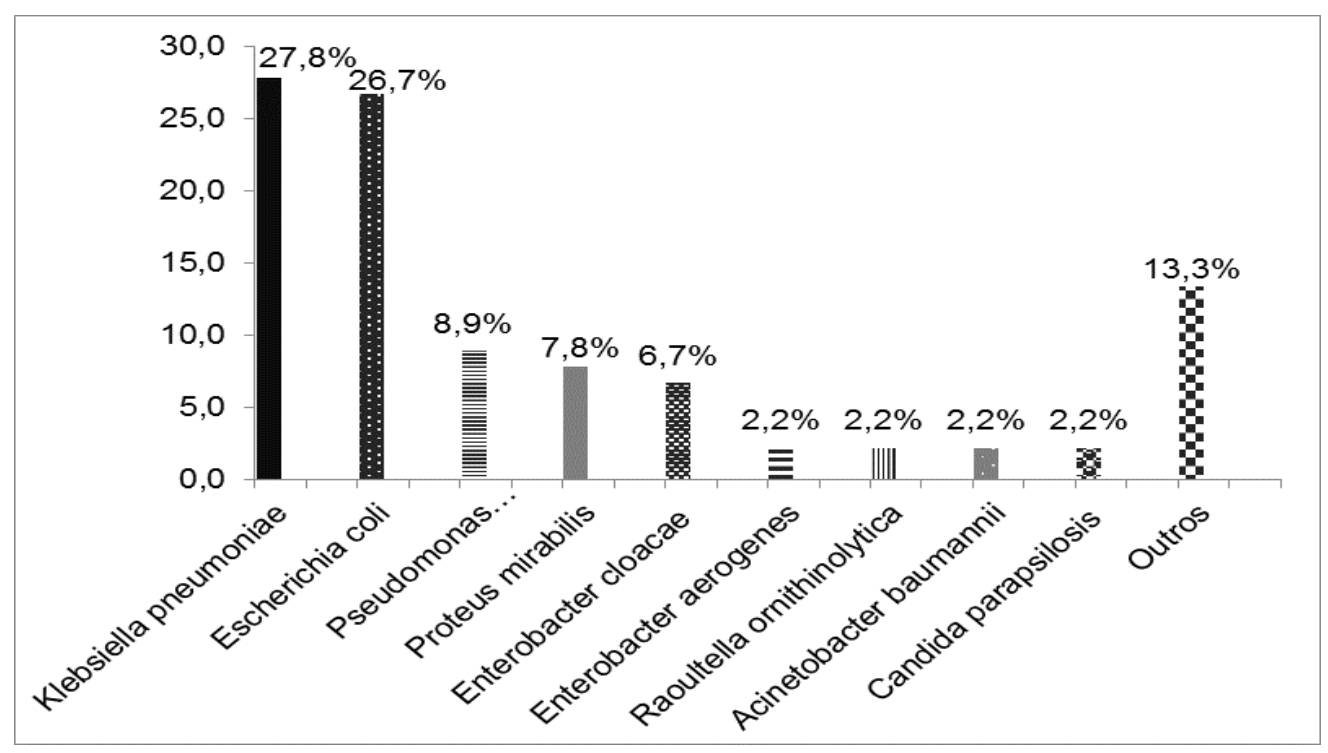

Figura 2 - Prevalência de microrganismos isolados em exames de urocultura em pacientes internos no HUAC.

Fonte: Pesquisadores

A Escherichia coli é uma das mais comuns bactérias Gram-negativas pertencentes à família Enterobacteriaceae, a qual segundo diversos autores é o membro mais importante nos quadros de infecção urinária na comunidade (MASSOLI et al., 2012; SAKRAN et al., 2015; KONCA et al., 2017).

O presente estudo corrobora com pesquisa anterior realizada no HUAC, no primeiro semestre de 2012, na qual se verificou o perfil epidemiológico da infecção nosocomial do trato urinário, onde predominaram $E$. coli e $K$. pneumoniae entre os microrganismos mais isolados, especialmente em indivíduos idosos (BARACUHY et al., 2013).

A frequência de microrganismos causando ITU em pacientes internos se assemelha aos estudos realizados por Silveira et al. (2010), Negromonte et al. (2015) e Das et al. (2017), nos quais E. coli e K. pneumoniae predominaram entre os isolados.

Quando a infecção é adquirida no ambiente hospitalar (pacientes internos), os agentes etiológicos são bastante diversificados, predominando as Enterobactérias, com redução na frequência de $E$. coli, e um crescimento de Proteus sp, Pseudomonas aeruginosa, Klebsiella sp., Enterobacter sp., Enterococcus faecalis e de fungos, com destaque para Candida sp (KSYCKI; NAMIAS, 2009; PELEG; HOOPER, 2010). 
O sexo feminino foi o mais acometido com 25 (73,5\%) dos casos de uroculturas positivas a nível ambulatorial. Com relação às faixas etárias, observou-se maior frequência com elevação da idade, principalmente após os cinquenta anos, em ambos os sexos (Figura 3 ).

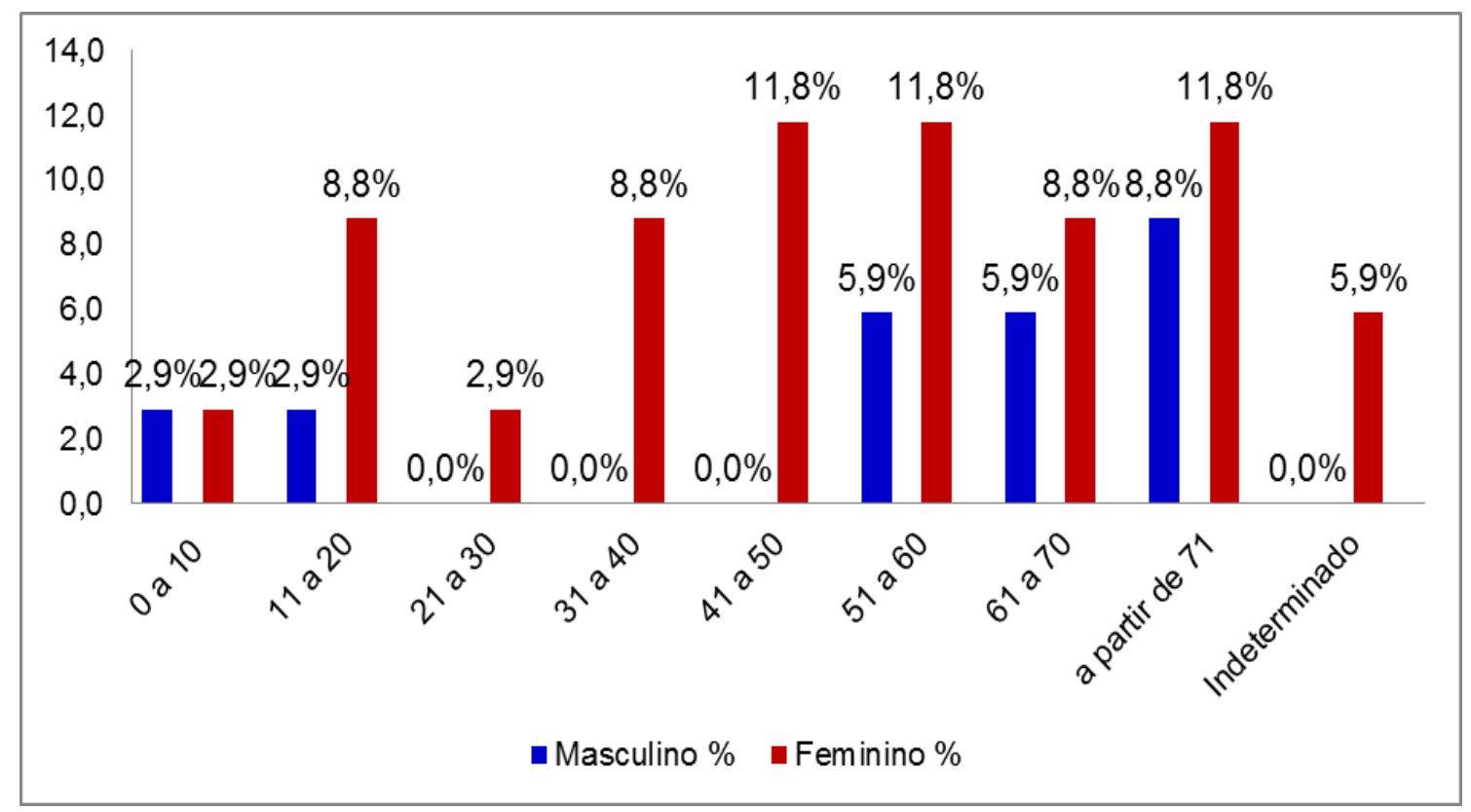

Figura 3 - Porcentagem de pacientes com uroculturas positivas a nível ambulatorial, distribuídos por faixa etária e sexo no HUAC.

Fonte: Pesquisadores

A maior frequência de infecção urinária, acometendo o sexo feminino, se repete entre os pacientes internos, atingindo 58 (64,4\%), estando à maioria destas mulheres entre 61 a 70 anos (16,7\%). Quanto ao sexo masculino, à faixa etária predominante foi a partir de 71 anos (10,0\%), conforme a Figura 4.

O presente estudo corrobora alguns anteriores, quanto a maior incidência de ITU em mulheres. Esse maior percentual para o sexo feminino pode ser explicado por diversos fatores, dentre eles a uretra ser mais curta e mais proximidade do ânus que a do homem (DE MOURA; 2011; DIAS; COELHO; DORIGON, 2011). 


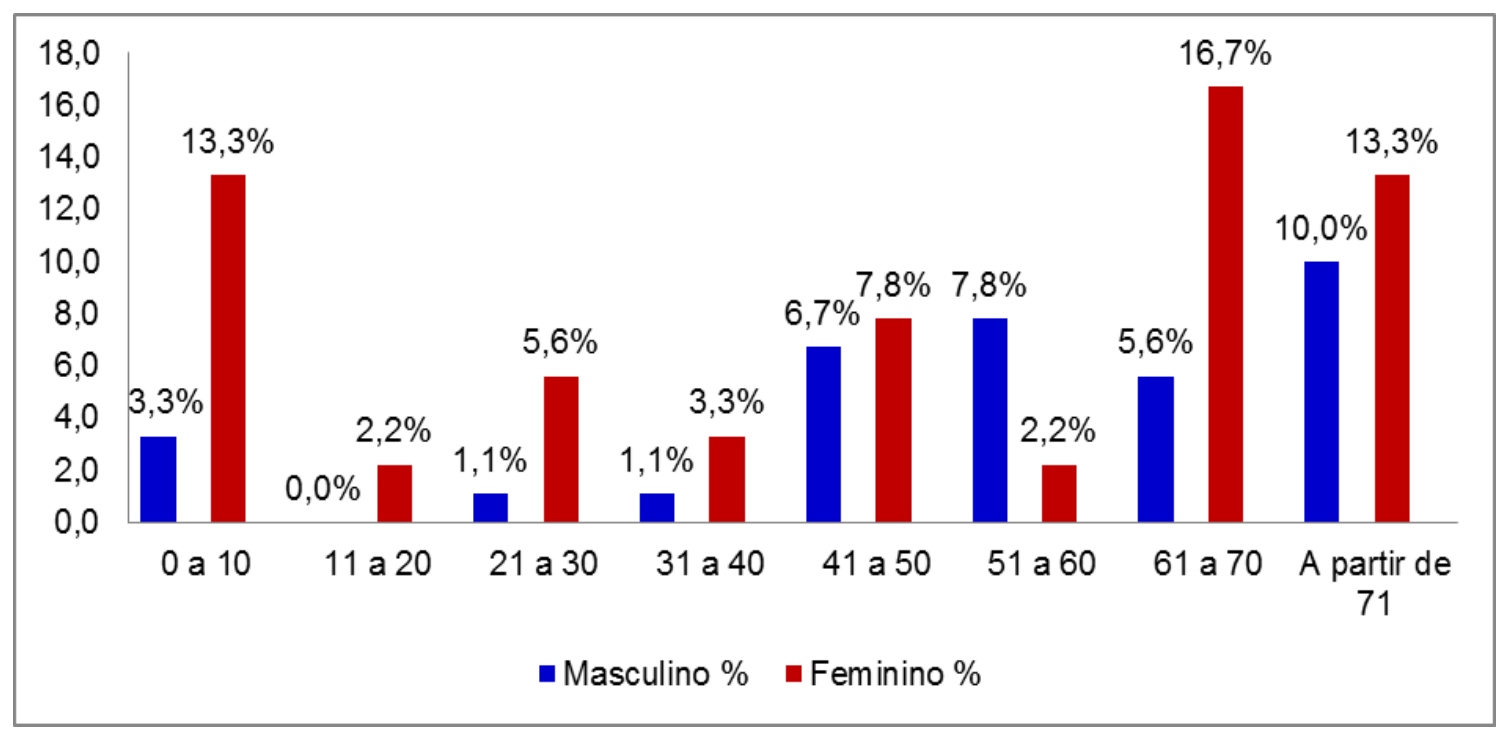

Figura 4 - Porcentagem de pacientes internos com uroculturas positivas, distribuídos por faixa etária e sexo no HUAC.

Fonte: Pesquisadores

No atual estudo, foram avaliados os perfis de sensibilidade a antimicrobianos para os microrganismos mais frequentes observados nos resultados de urocultura, ou seja, tanto para E. coli (ambulatorial) quanto para K. pneumoniae (nosocomial).

O perfil de sensibilidade aos antimicrobianos para Escherichia coli encontra-se na Tabela 1. Percebe-se que muitos antimicrobianos apresentaram bons resultados, com $100 \%$ de eficácia contra as cepas de E. coli, tais como amicacina, cefepima, ceftazidima e meropenem. Por outro lado, apresentou um perfil de resistência variável para alguns antimicrobianos testados.

$\mathrm{Na}$ avaliação dos testes de sensibilidade para $E$. coli isoladas de infecções oriundas da comunidade, a ampicilina apresentou a maior porcentagem de resistência (50\%), seguido do ácido nalidíxico (33,3\%), sulfametoxazol/trimetoprim (25\%), ciprofloxacina (19\%) e da levofloxacina $(16,7 \%)$.

Analisando-se vários estudos de caracterização do perfil de sensibilidade de $E$. coli à antimicrobianos, constatou-se elevada resistência a ampicilina como no presente estudo, Santana et al. (2012) 67\%, Rabello et al. (2011) 56\% e Massoli et al. (2012) 55\%. 
Tabela 1 - Perfil de sensibilidade a antimicrobianos para cepas de E. coli isoladas de pacientes ambulatoriais no HUAC

\begin{tabular}{|c|c|c|c|c|c|c|}
\hline \multirow[b]{2}{*}{ Antimicrobianos } & \multicolumn{2}{|c|}{ Sensíveis } & \multicolumn{2}{|c|}{ Resistentes } & \multicolumn{2}{|c|}{ Não testado } \\
\hline & $\mathrm{n}$ & $\%$ & $\mathrm{n}$ & $\%$ & $\mathrm{n}$ & $\%$ \\
\hline Amicacina & 21 & 100 & 0 & $\overline{0}$ & 0 & 0 \\
\hline $\begin{array}{l}\text { Amoxicilina/ Ácido } \\
\text { Clavulânico }\end{array}$ & 12 & 100 & 0 & 0 & 9 & 42,8 \\
\hline Ampicilina & 10 & 50 & 10 & 50 & 1 & 4,8 \\
\hline $\begin{array}{l}\text { Ampicilina/ } \\
\text { Sulbactam }\end{array}$ & 6 & 100 & 0 & 0 & 15 & 71,4 \\
\hline Aztreonam & 8 & 100 & 0 & 0 & 13 & 62 \\
\hline Cefalotina & 12 & 85,7 & 2 & 14,3 & 7 & 33,3 \\
\hline Cefepima & 21 & 100 & 0 & 0 & 0 & 0 \\
\hline Cefotaxima & 20 & 100 & 0 & 0 & 1 & 4,8 \\
\hline Cefoxitina & 19 & 90,5 & 2 & 9,5 & 0 & 0 \\
\hline Ceftazidima & 21 & 100 & 0 & 0 & 0 & 0 \\
\hline Ciprofloxacina & 17 & 81 & 4 & 19 & 0 & 0 \\
\hline Ertapenem & 20 & 95,2 & 1 & 4,8 & 0 & 0 \\
\hline Gentamicina & 20 & 100 & 0 & 0 & 1 & 4,8 \\
\hline Imipenem & 9 & 100 & 0 & 0 & 12 & 57,1 \\
\hline Meropenem & 21 & 100 & 0 & 0 & 0 & 0 \\
\hline Tigeciclina & 9 & 100 & 0 & 0 & 12 & 57,1 \\
\hline $\begin{array}{l}\text { Sulfametoxazol/ } \\
\text { Trimetoprim }\end{array}$ & 9 & 75 & 3 & 25 & 9 & 42,8 \\
\hline Ácido Nalidíxico & 8 & 66,7 & 4 & 33,3 & 9 & 42,8 \\
\hline Nitrofurantoína & 12 & 100 & 0 & 0 & 9 & 42,8 \\
\hline Levofloxacina & 10 & 83,3 & 2 & 16,7 & 9 & 42,8 \\
\hline
\end{tabular}

Santana et al. (2012), observaram que E. coli apresentou uma maior resistência ao ácido nalidíxico (82,5\%), enquanto Costa et al. (2010) demonstraram $(38,3 \%)$ de resistência, algo mais próximo ao observado no atual estudo (33,3\%). A resistência ao ácido nalidíxico pode ser justificada pelo fato deste antimicrobiano ter sido a primeira quinolona a ser obtida e por ter, muitas vezes de forma indiscriminada, usada bastante na pratica clínica, gerando uma resistência dos microrganismos.

Quanto a resistência a sulfametoxazol/trimetoprim observou-se taxa de $46,9 \%$ em estudo realizado por Koch et al. (2008), quase que o dobro do 
resultado demonstrado (25\%) de resistência no atual estudo. Massoli et al. (2012) também encontrou resultados mais elevados (56\%). Este último antimicrobiano foi durante muito tempo, uma das principais opções terapêuticas para tratamento de ITUs, no entanto, tem sido detectada uma diminuição na sua atividade, provavelmente em decorrência do uso indiscriminado, empírico e de outros fatores (RABELLO et al., 2011).

Costa et al. (2010) divulgaram uma alta incidência de resistência às quinolonas, relacionada a $E$. coli, com índice de $(21,3 \%)$, para ciprofloxacina. Resultado semelhante foi encontrado neste estudo $(19,1 \%)$.

Foram testadas 21 cepas, das quais uma única apresentou positividade para a produção de Betalactamases de Espectro Ampliado. A problemática das Enterobacteriaceae produtoras de ESBL, passou nos últimos anos a abranger não só as infecções nosocomiais, como também as infecções adquiridas na comunidade, representando atualmente um problema de saúde pública mundial (REULAND et al., 2016; KIM; YANG; KIM, 2017).

Quanto ao perfil de sensibilidade da Klebsiella pneumoniae, isolada de pacientes internos com infecção do trato urinário, verificou-se um maior grau de resistência aos antimicrobianos quando comparado ao perfil anterior para $E$. coli, conforme tabela 2.

Destaca-se que 11 (44\%) apresentaram positividade para a produção de Betalactamases de Espectro Ampliado (ESBL). A Escherichia coli e a Klebsiella pneumoniae são as espécies bacterianas mais comumente encontradas produzindo ESBL (DJUIKOUE et al., 2017).

Pasta et al. (2008), em estudo realizado no Hospital Universitário da Universidade Estadual de Londrina-Paraná, verificaram que $40 \%$ dos isolados de $K$. pneumoniae produziam ESBL, sendo $66,1 \%$ das amostras isoladas a partir da urina de pacientes internos.

Almeida (2013), em uma dissertação sobre resistência de Klebsiella pneumoniae a carbapenêmicos, demonstrou que todas as amostras testadas foram resistentes à ciprofloxacina, cefoxitina, cefotaxima e cefepima, bem como altas taxas de resistência aos carbapenêmicos: 100\% para ertapenem, $68,2 \%$ para meropenem e $45,4 \%$ para imipenem. Contudo, nem todas as amostras analisadas eram provenientes de urina. No atual estudo, foram 
obtidos menores índices de resistência a ciprofloxacina, a cefotaxima e a cefepima, e quanto aos carbapenens, o ertapenem obteve valor inferior $(60,9 \%)$ e o meropenem diminuído em comparação a este estudo. No entanto, vale ressaltar que na presente pesquisa, outras enfermidades estariam envolvidas além das ITUs.

Tabela 2 - Perfil de Sensibilidade a Antimicrobianos para cepas de $K$. pneumoniae isoladas de pacientes internos no HUAC

\begin{tabular}{|c|c|c|c|c|c|c|}
\hline \multirow[b]{2}{*}{ Antimicrobianos } & \multicolumn{2}{|c|}{ Sensíveis } & \multicolumn{2}{|c|}{ Resistentes } & \multicolumn{2}{|c|}{ Não testado } \\
\hline & $\mathrm{n}$ & $\%$ & $\mathrm{n}$ & $\%$ & $\mathrm{n}$ & $\%$ \\
\hline Amicacina & 24 & 96 & 1 & 4 & 0 & 0 \\
\hline $\begin{array}{l}\text { Amoxicilina/ Ácido } \\
\text { Clavulânico }\end{array}$ & 4 & 40 & 6 & 60 & 15 & 60 \\
\hline Ampicilina & 1 & 4 & 24 & 96 & 0 & 0 \\
\hline $\begin{array}{l}\text { Ampicilina/ } \\
\text { Sulbactam }\end{array}$ & 5 & 36 & 9 & 64 & 11 & 44 \\
\hline Aztreonam & 5 & 33,3 & 10 & 66,7 & 10 & 40 \\
\hline Cefalotina & 12 & 66,7 & 6 & 33,3 & 3 & 12 \\
\hline Cefepima & 11 & 45,8 & 13 & 54,2 & 1 & 4 \\
\hline Cefotaxima & 9 & 37,5 & 15 & 62,5 & 1 & 4 \\
\hline Cefoxitina & 13 & 72,2 & 5 & 27,8 & 7 & 28 \\
\hline Ceftazidima & 9 & 39,1 & 14 & 60,9 & 2 & 8 \\
\hline Ciprofloxacina & 10 & 40 & 15 & 60 & 0 & 0 \\
\hline Ertapenem & 9 & 39,1 & 14 & 60,9 & 2 & 8 \\
\hline Gentamicina & 10 & 40 & 15 & 60 & 0 & 0 \\
\hline Imipenem & 14 & 93,3 & 1 & 6,7 & 10 & 40 \\
\hline Meropenem & 24 & 96 & 1 & 4 & 0 & 0 \\
\hline Tigeciclina & 8 & 66,7 & 4 & 33,3 & 13 & 52 \\
\hline $\begin{array}{l}\text { Sulfametoxazol/ } \\
\text { Trimetoprim }\end{array}$ & 2 & 20 & 8 & 80 & 15 & 60 \\
\hline Ácido Nalidíxico & 3 & 30 & 7 & 70 & 15 & 60 \\
\hline Nitrofurantoína & 2 & 25 & 6 & 75 & 17 & 68 \\
\hline Levofloxacina & 4 & 40 & 6 & 60 & 15 & 60 \\
\hline $\begin{array}{l}\text { Piperacilina/ } \\
\text { Tazobactam }\end{array}$ & 10 & 100 & 0 & 0 & 15 & 60 \\
\hline
\end{tabular}


Uma menor resistência à cefoxitina costuma ser associada ao fato desta não ser frequentemente utilizada na prática clínica para o tratamento de infecções por Klebsiella spp. (PASTA et al., 2008).

Oliveira et al. (2011) em estudo realizado em um hospital da cidade de Natal-RN demonstraram que ocorreu uma variação anual e uma tendência para o crescimento da resistência aos antimicrobianos frente à Klebsiella spp. durante o período de 10 anos. Constatou-se ainda que, praticamente todos os antimicrobianos testados apresentaram aumento da resistência aos agentes antimicrobianos com o passar dos anos.

Cyrino e Stuchi (2016) apontam como principal fator de risco para aquisição de infecção urinária a utilização de sonda vesical de demora, sendo a duração da cateterização relevante para ocorrência de ITU.

\section{Conclusão}

A quantidade de uroculturas positivas, sexo e idade mais frequentes dos pacientes, principais espécies isoladas ( $E$. coli e $K$. pneumoniae) foram semelhantes a outros trabalhos anteriormente divulgados.

Chama atenção a grande resistência das bactérias $E$. coli e $K$. pneumoniae, especialmente a ampicilina, desestimulando o uso empírico deste fármaco tanto, em infecções comunitárias, quanto em ITU de origem hospitalar.

Outro fator preocupante observado foi o elevado número de cepas de $K$. pneumoniae produtoras de ESBL, respaldando a importância do antibiograma para a melhor escolha terapêutica. A realização dos testes de sensibilidade a antimicrobianos permite uma terapia mais eficaz, evitando o uso inapropriado, que geralmente aumenta a resistência microbiana.

O conhecimento da epidemiologia das infecções do trato urinário e a implantação de protocolos terapêuticos para utilização de antimicrobianos, buscando uma padronização do uso, juntamente com os resultados do antibiograma, pode permitir uma redução da resistência aos agentes antimicrobianos.

\section{Referências}


ALLGAYER, Natacha; SCHIRMER, Helena; CASTELAN, Jussara Amaro. Concordância dos resultados do sistema BD Phoenix com provas bioquímicas manuais na identificação de Enterobactérias em amostras clínicas. Clinical \& Biomedical Research, v. 35, n. 1, 2015.

ALMEIDA, Viviene Vieira Prado. Infecções por Klebsiella pneumoniae resistente aos carbapenêmicos em hospital de nível terciario: epidemiologia e caracterização. 2013. 66 f. Dissertação (Mestrado em Ciências da Saúde) - Faculdade de Medicina da Universidade Federal de Uberlândia, Uberlândia, 2013.

BARACUHY, Ygor Paiva Schiel et al. Perfil epidemiológico da infecção nosocomial do trato urinário em hospital universitário de Campina Grande (PB). Arquivos Brasileiros de Ciências da Saúde, v. 38, n. 3, p. 146-152, abr./set. 2013.

BRASIL, Agência Nacional de Vigilância Sanitária. Educação em Saúde Infecção do Trato Urinário, 2002. Disponível em: http://www.anvisa.gov.br Acesso em: 3 jul. 2013.

COSTA, Fabiana Cristina. Prevalência e Perfil de Suscetibilidade a Antimicrobianos de Microrganismos Isolados em Urocultura no Período de Janeiro a Dezembro de 2007, em Hospital Municipal de lbirité/MG. 2009. 80 f. Monografia (Especialização em Microbiologia Aplicada às Ciências da Saúde) - Universidade Federal de Minas Gerais, Belo Horizonte, 2009.

COSTA, Larissa Chaves et al. Infecções urinárias em pacientes ambulatoriais: prevalência e perfil de resistência aos antimicrobianos. Revista Brasileira de Análises Clínicas, v. 42, n. 3, p. 175-180, 2010.

CYRINO, Ana Cristina Teixeira; STUCHI, Rosamary Aparecida Garcia. Infecção do trato urinário em um hospital de uma cidade no interior de Minas Gerais. Revista de Enfermagem da UFJF, v. 1, n. 1, 2016. 
DAS, R. et al. Clinical risk factors, bacterial aetiology, and outcome of urinary tract infection in children hospitalized with diarrhoea in Bangladesh. Epidemiology \& Infection, v. 145, n. 5, p. 1018-1024, dez. 2016/abr. 2017.

DE MOURA, Lorena Brandhuber. A incidência de infecções urinárias causadas por E. coli. Olhar Científico, v. 1, n. 2, p. 411-426, 2011.

DIAS, Ilo Odilon Villa; COELHO, Alessandra Mello; DORIGON, Ionara. INFECÇÃO DO TRATO URINÁRIO EM PACIENTES AMBULATORIAIS: PREVALÊNCIA E PERFIL DE SENSIBILIDADE AOS ANTIMICROBIANOS EM ESTUDO REALIZADO DE 2009 A 2012. Saúde (Santa Maria), v. 41, n. 1, p. 209-218, 2015.

DJUIKOUE, Ingrid Cecile et al. Prevalence of CTX-M beta-Lactamases in Escherichia coli from community-acquired urinary tract infections and associated risk factors among women in Cameroon. Journal of Epidemiological Research, v. 3, n. 1, p. 51-56, dez. 2016/ jan. 2017.

HACHUL, Maurício et al. Infecção do trato urinário complicada. RBM Revista Brasileira de Medicina, v. 72, n. 9, p. 383-7, 2015

HEILBERG, Ita Pfeferman; SCHOR, Nestor. Abordagem Diagnóstica e Terapêutica na Infecção do Trato Urinário: ITU. Revista da Associação Médica Brasileira, v. 49, n., p. 109-116, 2003.

KIM, Yun Hee; YANG, Eun Mi; KIM, Chan Jong. Infecção do trato urinário causada por bactérias produtoras de $\beta$-lactamases de espectro ampliado adquiridas na comunidade em neonatos. Jornal de Pediatria, v. 93, n. 3, p. 260-266, 2017. 
$\mathrm{KOCH}$, Camila Ribeiro et al. Resistência antimicrobiana dos uropatógenos em pacientes ambulatoriais, 2000-2004. Revista da Sociedade Brasileira de Medicina Tropical, v. 4, n. 3, p. 277-281, 2008.

KONCA, Capan et al. Antibacterial resistance patterns of pediatric communityacquired urinary infection: Overview. Pediatrics international, v. 59, n.3, p. 309-315, nov. 2016 /mar. 2017.

KONEMAN, Elmer W. et al. Diagnóstico Microbiológico - Texto e atlas colorido. 6. ed. Rio de Janeiro: Guanabara Koogan, 2008.

KSYCKI, Michael F.; NAMIAS, Nicholas. Nosocomial Urinary Tract Infection. Surgical Clinics of North America, v. 89, n. 2, p. 475-81, 2009.

MIRANDA, Anna Leticia et al. Resultados da implementação de um protocolo sobre a incidência de Infecção do Trato Urinário em Unidade de Terapia Intensiva. Revista Latino-Americana de Enfermagem, v. 24, p. 1-9, 2016.

NEGROMONTE, Gustavo Ramalho Pessoa et al. Study of variables involved in hospital-acquired urinary tract infections at a university hospital. Revista da Sociedade Brasileira de Clínica Médica, v. 13, n. 2, p. 90-93, abr./jun. 2015.

OLIVEIRA, Claudio Bruno Silva et al. Frequência e perfil de resistência de Klebsiella spp. em um hospital universitário de Natal/RN durante 10 anos. Jornal Brasileiro de Patologia e Medicina Laboratorial, v. 47, n. 6, p. 589594, 2011.

PASTA, Angelo Augusto Chiachia et al. Prevalência e perfil de susceptibilidade antimicrobiana em cepas de Klebsiella pneumoniae produtoras de $\beta$ lactamases de espectro estendido (ESBL), isoladas de pacientes do Hospital universitário/UEL. Revista Brasileira de Análises Clínicas, v. 40, n. 2, p. 13741, 2008. 
PELEG, Anton Y.; HOOPER, David C. Hospital-Acquired Infections Due to Gram-Negative Bacteria. New England Journal of Medicine, v. 362, n. 19, p.1804-1813, 2010.

POLIT, Denise F.; BECK, Cheryl Tatano; HUNGLER, Bernadette P. Fundamentos de Pesquisa em Enfermagem, Métodos, Avaliação e Utilização, 5. ed. Porto Alegre: Artmed, 2004.

RABELLO, Rodolfo Augusto Araújo et al. Caracterização do perfil de resistência à fluoroquinolonas, Patos de Minas (MG). Revista Brasileira de Análises Clínicas, v. 43, n. 2, p. 110-115, 2011.

REULAND, E. A. et al. Prevalence and risk factors for carriage of ESBLproducing Enterobacteriaceae in Amsterdam. Journal of Antimicrobial Chemotherapy, v. 71, n. 4, p. 1076-1082, 2016.

ROSA, Rossana et al. Antimicrobial resistance in urinary tract infections at a large urban ED: Factors contributing to empiric treatment failure. The American Journal of Emergency Medicine, v. 35, n.3, p. 397-401, marc. 2017.

SAKRAN, Waheeb et al. Community-Acquired Urinary Tract Infection in Hospitalized Children: Etiology and Antimicrobial Resistance. A Comparison Between First Episode and Recurrent Infection. Clinical pediatrics, v. 54, p. 479-483, 2015.

SANTANA, Tatiana Cristina Fonseca Soares et al. Perfil de resistência de Escherichia coli e Klebsiella spp isoladas de urocultura de comunidade do município de São Luis-MA no período de 2005-2008. Revista de Patologia Tropical, v. 4, n. 3, p. 295-303, jul./set. 2012.

\section{Agradecimentos}

Ao Hospital Universitário Alcides Carneiro por ter gentilmente autorizado e cedido os dados necessários à pesquisa. 